\title{
Condensation of the roots of real random polynomials on the real axis
}

\author{
Grégory Schehr ${ }^{1}$, Satya N. Majumdar ${ }^{2}$ \\ ${ }^{1}$ Laboratoire de Physique Théorique, Université de Paris-Sud, 91405 Orsay France \\ ${ }^{2}$ Laboratoire de Physique Théorique et Modèles Statistiques, Université Paris-Sud, \\ Bât. 100, 91405 Orsay Cedex, France
}

\begin{abstract}
We introduce a family of real random polynomials of degree $n$ whose coefficients $a_{k}$ are symmetric independent Gaussian variables with variance $\left\langle a_{k}^{2}\right\rangle=e^{-k^{\alpha}}$, indexed by a real $\alpha \geq 0$. We compute exactly the mean number of real roots $\left\langle N_{n}\right\rangle$ for large $n$. As $\alpha$ is varied, one finds three different phases. First, for $0 \leq \alpha<1$, one finds that $\left\langle N_{n}\right\rangle \sim\left(\frac{2}{\pi}\right) \log n$. For $1<\alpha<2$, there is an intermediate phase where $\left\langle N_{n}\right\rangle$ grows algebraically with a continuously varying exponent, $\left\langle N_{n}\right\rangle \sim \frac{2}{\pi} \sqrt{\frac{\alpha-1}{\alpha}} n^{\alpha / 2}$. And finally for $\alpha>2$, one finds a third phase where $\left\langle N_{n}\right\rangle \sim n$. This family of real random polynomials thus exhibits a condensation of their roots on the real line in the sense that, for large $n$, a finite fraction of their roots $\left\langle N_{n}\right\rangle / n$ are real. This condensation occurs via a localization of the real roots around the values $\pm \exp \left[\frac{\alpha}{2}\left(k+\frac{1}{2}\right)^{\alpha-1}\right], 1 \ll k \leq n$.
\end{abstract}




\section{Introduction}

Since the early work of Bloch and Pólya [1] in the 30's, the study of random algebraic equations has now a long story [2, 3]. In the last few years, it attracted a renewed interest in the context of probability and number theory [4], as well as in the field of quantum chaos [5]. Recently, we showed that there are also interesting connections between random polynomials and persistence properties of physical systems [6, 7].

Here we consider real random polynomials, i.e. polynomials with real random coefficients, of degree $n$. While these polynomials have exactly $n$ roots in the complex plane, the number of roots on the real line $N_{n}$ is a random variable. One would like to characterize the statistics of this random variable and a natural question is thus : what is the mean number $\left\langle N_{n}\right\rangle$ of real roots and how does it behave with $n$ for large $n$ [4]? This question has been widely studied in the past for Kac's polynomials $K_{n}(x)=\sum_{k=0}^{n} a_{k} x^{k}$ where $a_{k}$ are independent and identically distributed (i.i.d.) random variables of finite variance $\left\langle a_{k}^{2}\right\rangle=\sigma^{2}$. In that case it is well known that $\left\langle N_{n}\right\rangle \sim \frac{2}{\pi} \log n$, independently of $\sigma$. This result was first obtained by Kac [8] for Gaussian random variables and it was later shown to hold also for a wider class of distributions of the coefficients $a_{k}$ [2, 3]. Interesting generalizations of Kac's polynomials have been studied in the literature where $a_{k}$ are independent Gaussian variables but non identical, such that $\left\langle a_{k}^{2}\right\rangle=k^{d-2}$, where $d>0$ is a real number, leading to $\left\langle N_{n}\right\rangle \sim \pi^{-1}(1+\sqrt{d / 2}) \log n$ [7, 9]. Given the robustness of this asymptotic logarithmic behavior of $\left\langle N_{n}\right\rangle$, it is natural to search for random polynomials for which $\left\langle N_{n}\right\rangle$ increases faster than $\log n$, for instance algebraically.

One such instance is provided by the real Weyl polynomials $W_{n}(x)$ defined by

$$
W_{n}(x)=\sum_{k=0}^{n} \epsilon_{k} \frac{x^{k}}{\sqrt{k !}},
$$

where $\epsilon_{k}$ are i.i.d. random variables of zero mean and unit variance. Thus here, $a_{k}=\epsilon_{k} / \sqrt{k !}$ and the variance is $\left\langle a_{k}^{2}\right\rangle=1 / k$ !, which for large $k$ behaves as $\left\langle a_{k}^{2}\right\rangle \propto e^{-k \log k}$. For these real polynomials in Eq. (1), it is known that $\left\langle N_{n}\right\rangle \propto n^{1 / 2}$. For instance, in the special case where $\epsilon_{k}$ are Gaussian random variables of unit variance, one has $\left\langle N_{n}\right\rangle \sim \frac{2}{\pi} \sqrt{n}$ [7, [10]. Another interesting and intriguing instance of real random polynomials was introduced a long time ago by Littlewood and Offord [11] who studied the random polynomials $L_{n}(x)$ given by

$$
L_{n}(x)=\frac{1}{2}+\sum_{k=1}^{n} \epsilon_{k} \frac{x^{k}}{(k !)^{k}},
$$

where $\epsilon_{k}= \pm 1$ with equal probability. Thus in this case $a_{k}=\epsilon_{k} /(k !)^{k}$ and the variance is $\left\langle a_{k}^{2}\right\rangle=1 /(k !)^{2 k}$, which behaves for large $k$ as $\left\langle a_{k}^{2}\right\rangle \propto e^{-2 k^{2} \log k}$. Using algebraic methods, they showed that such polynomials $L_{n}(x)$ have all their roots real and therefore $\left\langle N_{n}\right\rangle=n$.

We thus have here two examples of real random polynomials in Eq. (1) and Eq. (2) where, at variance with Kac's polynomials, $\left\langle N_{n}\right\rangle$ grows algebraically with $n$. In the second example (2), the number of real roots is "macroscopic" in the sense that, for 


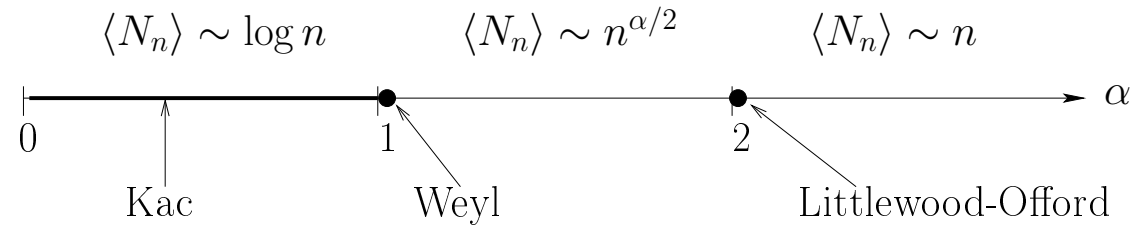

Figure 1. Asymptotic behavior of the mean number of real roots $\left\langle N_{n}\right\rangle$ of $P_{n}(x)$ in Eq. (3) as a function of $\alpha$. These polynomials exhibit a condensation of their roots on the real axis for $\alpha \geq 2$.

large $n$, there is a finite fraction $\left\langle N_{n}\right\rangle / n$ of the roots which are on the real axis. For $L_{n}(x)$ in Eq. (2) this fraction is exactly one. We thus say that there is a condensation of the roots on the real line, similar to a Bose-Einstein condensation where a finite fraction of the particles of a quantum-mechanical system (Bosons) condense into the lowest energy level. In the case of random polynomials, the roots play the role of the particles and the equivalent of the ground state is the real line.

The purpose of this paper is to understand what types of polynomials lead to this condensation phenomenon. Of course, it is very difficult to address this question for any random coefficients $a_{k}$. However, guided by the two examples above in Eq. (1) and Eq. (2), and in particular by the large $k$ behavior of $\left\langle a_{k}^{2}\right\rangle$, we introduce a family of random polynomials $P_{n}(x)$ indexed by a real $\alpha \geq 0$ defined by

$$
P_{n}(x)=\sum_{k=0}^{n} a_{k} x^{k},\left\langle a_{k}^{2}\right\rangle=e^{-k^{\alpha}},
$$

where $a_{k}$ are real independent Gaussian random variables of zero mean. While $\alpha=0$ corresponds to Kac's polynomials, we recall that, for $W_{n}(x)$ in Eq. (11), $\left\langle a_{k}^{2}\right\rangle \propto e^{-k \log k}$ and for $L_{n}(x)$ in Eq. (2), $\left\langle a_{k}^{2}\right\rangle \propto e^{-2 k^{2} \log k}$. Therefore, due to the extra logarithmic factor, these random polynomials are not exactly of the form introduced above (3). However, for $\alpha \rightarrow 1^{+}$, one expects to recover the behavior of $W_{n}(x)$ in Eq. (1) while for $\alpha \rightarrow 2^{+}$, one expects $P_{n}(x)$ to behave similarly to $L_{n}(x)$ in Eq. (2) : this is depicted schematically in Fig. 1 .

Our main results can be summarized as follows. As $\alpha \geq 0$ is varied one finds three different phases. The first phase corresponds to $0 \leq \alpha<1$, where one finds that $\left\langle N_{n}\right\rangle \sim(2 / \pi) \log n$. In the second one, corresponding to $1<\alpha<2$, one has $\left\langle N_{n}\right\rangle \sim \frac{2}{\pi} \sqrt{\frac{\alpha-1}{\alpha}} n^{\alpha / 2}$. And in the third phase, for $\alpha>2$, one finds $\left\langle N_{n}\right\rangle \sim n$. The condensation of the roots on the real axis thus happens for $\alpha \geq 2$ and as one increases $\alpha$, the condensation transition sets in at the critical value $\alpha_{c}=2$. Furthermore, one finds that these real roots condense into a quasi-periodic structure such that there is, on average, one root in the interval $\left[-x_{m+1},-x_{m}\right] \cup\left[x_{m}, x_{m+1}\right]$, with $x_{m}=e^{\frac{\alpha}{2} m^{\alpha-1}}$, with $1 \ll m<n$. These different behaviors are summarized in Fig. 1, By analogy with phase transitions of statistical systems the case $0<\alpha<1$ can be considered as a high-temperature phase whereas $\alpha>2$ corresponds to the low-temperature (ordered) phase. Roughly speaking, one can consider our results as an interesting example where the transition from the high temperature where $\left\langle N_{n}\right\rangle \propto \log n$ (governed by a " $\alpha=0$ 
fixed point") to the low temperature phase where $\left\langle N_{n}\right\rangle \propto n$ (governed by " $\alpha=\infty$ " fixed point) happens through a marginal phase, for $1<\alpha<2$, where $\left\langle N_{n}\right\rangle \sim n^{\phi}$ with an exponent $\phi=\alpha / 2$ which depends continuously on $\alpha$.

The paper is organized as follows. In section 2, we describe the general framework to compute the local density of real roots, which directly leads to $\left\langle N_{n}\right\rangle$. In section 3 to 6 we then analyse separately the cases $0 \leq \alpha<1, \alpha<2, \alpha>2$ and the "critical case" $\alpha=2$. In section 7 , we give a qualitative argument to explain the condensation transition occurring at $\alpha_{c}=2$ before we conclude in section 8. The Appendix contains some useful technical details.

\section{General framework}

First we notice that given that $P_{n}(x)$, as a function of $x$, is a Gaussian process, it is completely characterized by its two-point correlation function $C_{n}(x, y)$

$$
C_{n}(x, y)=\left\langle P_{n}(x) P_{n}(y)\right\rangle=\sum_{k=0}^{n} e^{-k^{\alpha}} x^{k} y^{k},
$$

where we used the notation $\langle\ldots\rangle$ to denote an average over the random variables $a_{k}$. A central object involved in the calculation of $\left\langle N_{n}\right\rangle$ is $\rho_{n}(x)$, the mean density of real roots at point $x$. If we denote $\lambda_{1}, \lambda_{2}, \ldots, \lambda_{p}$ the $p$ real roots (if any) of $P_{n}(x)$, one has $\delta\left(P_{n}(x)\right)=\sum_{i=1}^{p} \delta\left(x-\lambda_{i}\right) /\left|P_{n}^{\prime}\left(\lambda_{i}\right)\right|$ such that $\rho_{n}(x)$ can be written as

$$
\begin{aligned}
\rho_{n}(x) & =\sum_{i=1}^{p}\left\langle\delta\left(x-\lambda_{i}\right)\right\rangle=\left\langle\left|P_{n}^{\prime}(x)\right| \delta\left(P_{n}(x)\right)\right. \\
& =\int_{-\infty}^{\infty} d y|y|\left\langle\delta\left(P_{n}^{\prime}(x)-y\right) \delta\left(P_{n}(x)\right)\right\rangle .
\end{aligned}
$$

Under this form (5), one observes that the computation of the mean density involves the joint distribution of the polynomial $P_{n}(x)$ and its derivative $P_{n}^{\prime}(x)$ which is simply a bivariate Gaussian distribution. After Gaussian integration over $y$, one obtains

$$
\begin{aligned}
& \rho_{n}(x)=\frac{\sqrt{c_{n}(x)\left(c_{n}^{\prime}(x) / x+c_{n}^{\prime \prime}(x)\right)-\left[c_{n}^{\prime}(x)\right]^{2}}}{2 \pi c_{n}(x)}, \\
& c_{n}(x)=C_{n}(x, x)=\sum_{k=0}^{n} e^{-k^{\alpha}} x^{2 k} .
\end{aligned}
$$

This formula (66) can be written in a very compact way [4]

$$
\rho_{n}(x)=\left.\frac{1}{\pi} \sqrt{\partial_{u} \partial_{v} \log C_{n}(u, v)}\right|_{u=v=x} .
$$

Given that the random coefficients $a_{k}$ are drawn from a symmetric distribution, we can restrict our study of $\rho_{n}(x)$ on $\mathbb{R}^{+}$from which one obtains the mean number of real roots $\left\langle N_{n}\right\rangle$ as

$$
\left\langle N_{n}\right\rangle=2 \int_{0}^{\infty} \rho_{n}(x) d x
$$


An important change of variable. We will see below that it is useful to consider these polynomials $P_{n}(x)$ in terms of another variable $Y$ defined as

$$
Y=\left(\frac{2}{\alpha} \log x\right)^{\frac{1}{\alpha-1}} .
$$

We denote $\hat{\rho}_{n}(Y)$ the mean density of the real roots in terms of this new variable such that one has also $\left\langle N_{n}\right\rangle=\int_{0}^{\infty} \hat{\rho}_{n}(Y) d Y$. For $0<\alpha<1$ we will see that, for large $n$, most of the real roots of $P_{n}$ are located close to $Y=n$ while for $\alpha>1$, the density extends over the whole interval $Y \in[1, n]$. This change of variable (9) is motivated by the following analysis.

First we notice that $C_{n}(x, y)=\sum_{k=0}^{n} e^{-k^{\alpha}} x^{k} y^{k}$ in Eq. (44) is of the form $C_{n}(x, y)=$ $c_{n}(\sqrt{x y})$. Anticipating a saddle point analysis, one writes $c_{n}(x)$ as

$$
c_{n}(x)=\sum_{k=0}^{n} e^{-k^{\alpha}} x^{2 k}=\sum_{k=0}^{n} \exp (-\phi(k, x)), \phi(k, x)=k^{\alpha}-2 k \log x .
$$

Although $\phi(k, x)$ is defined for integers $k=0,1,2, \cdots, n$, it is readily extended to the real axis and denoted $\phi(u, x)=u^{\alpha}-2 u \log x$ for $u \in \mathbb{R}^{+}$. The behavior of $c_{n}(x)$ is essentially governed by the behavior of $\phi(u, x)$ as a function of $u$ (and fixed $x$ ). In particular, for $\alpha<1, \phi(u, x)$ has a single maximum while for $\alpha>1$, it has a single minimum for $u=u^{*}(x)$ given by

$$
\begin{aligned}
& \partial_{u} \phi\left(u^{*}(x), x\right)=0, \partial_{u}^{2} \phi\left(u^{*}(x), x\right)=\alpha(\alpha-1) u^{*}(x)^{\alpha-2}>0, \\
& u^{*}(x)=\left(\frac{2}{\alpha} \log x\right)^{\frac{1}{\alpha-1}} .
\end{aligned}
$$

The new variable $Y$ introduced above in Eq. (9) is thus precisely $Y=u^{*}(x)$. As a consequence, the density behaves quite differently in both cases $\alpha<1$ and $\alpha>1$.

For $\alpha<1$, most of the real roots on $\mathbb{R}^{+}$are located in $[1, \infty]$. For fixed $x>1$, $\phi(u, x)$ as a function of $u$ in the interval $[0, n]$ has a global minimum for $u=n$. Therefore, the sum entering in the expression of $c_{n}(x)$ in Eq. (10) will be dominated by the terms with $k \sim n$. The expansion of $\phi(k, x)$ in Taylor series around $k=n$ yields

$$
\begin{aligned}
\phi(k, x) & =\phi(n, x)+(k-n)\left(\alpha n^{\alpha-1}-2 \log x\right)+\cdots \\
& =(1-\alpha) n^{\alpha}-k\left(\alpha n^{\alpha-1}-2 \log x\right)+\cdots,
\end{aligned}
$$

where the higher order terms can be neglected in the large $n$ limit because $\partial^{j} \phi(n, x) / \partial u^{j}=\mathcal{O}\left(n^{\alpha-j}\right)$ for $j \geq 2$. Thus, for $\alpha<1$ one has

$$
c_{n}(x) \sim e^{-(1-\alpha) n^{\alpha}} \sum_{k=0}^{n}\left(x e^{-\frac{\alpha}{2} n^{\alpha-1}}\right)^{2 k},
$$

which, in terms of the rescaled variable $\tilde{x}=x e^{-\frac{\alpha}{2} n^{\alpha-1}}$, is the correlator of Kac's polynomials. From this observation (13), one can straightforwardly obtain the mean number of real roots $\left\langle N_{n}\right\rangle$, this will be done in section 3 .

For $\alpha>1$, the situation is quite different and in that case, $\phi(u, x)$ has a single minimum for $u=u^{*}(x)=\left(\frac{2}{\alpha} \log x\right)^{\frac{1}{\alpha-1}}$ (11). Besides, we will see below that the 
main contribution to $\left\langle N_{n}\right\rangle$ on $\mathbb{R}^{+}$comes from the interval $1<x<\exp \left(\frac{\alpha}{2} n^{\alpha-1}\right)$ where $1<u^{*}(x)<n$. In that case the sum entering in the definition of $c_{n}(x)$ in Eq. (10) is indeed dominated by $k \sim u^{*}(x)$ and $c_{n}(x)$ can be evaluated by a saddle point calculation. For this purpose, one obtains after some algebra explained in the Appendix, a convenient expression of $\rho_{n}(x)$ as

$$
\rho_{n}(x)=\frac{1}{\pi x}\left(\frac{\sum_{k=0}^{n}\left(k-u^{*}(x)\right)^{2} e^{-\phi(k, x)}}{\sum_{k=0}^{n} e^{-\phi(k, x)}}-\left[\frac{\sum_{k=0}^{n}\left(k-u^{*}(x)\right) e^{-\phi(k, x)}}{\sum_{k=0}^{n} e^{-\phi(k, x)}}\right]^{2}\right)^{\frac{1}{2}}
$$

which is the starting point of our analysis for $\alpha>1$. For $1<x<\exp \left(\frac{\alpha}{2} n^{\alpha-1}\right)$, one has $u^{*}(x)<n$ so that the sums over $k$ in Eq. (14) are dominated by $k \sim u^{*}(x)$. The Taylor expansion of $\phi(k, x)$ around this minimum reads

$$
\phi(k, x)=\phi\left(u^{*}(x), x\right)+\sum_{j=2}^{\infty} \frac{\alpha(\alpha-1) \ldots(\alpha-j+1)}{j !}\left(k-u^{*}(x)\right)^{j}\left[u^{*}(x)\right]^{\alpha-j} .(15)
$$

For large $x, u^{*}(x) \propto(\log x)^{1 /(\alpha-1)}$ is also large so that, to leading order in $x$, one can retain only the term corresponding to $j=2$ in the Taylor expansion in Eq. (15). This yields, for large $x$

$$
\begin{aligned}
& \sum_{k=0}^{n} g\left(k-u^{*}(x)\right) \exp (-\phi(k, x)) \\
& \sim e^{-\phi\left(u^{*}(x), x\right)} \sum_{k=0}^{n} g\left(k-u^{*}(x)\right) \exp \left[-\frac{\alpha(\alpha-1)}{2}\left(k-u^{*}(x)\right)\left[u^{*}(x)\right]^{\alpha-2}\right],
\end{aligned}
$$

with $g(z)=z$ or $g(z)=z^{2}$ as in Eq. (14). For later purpose it is useful to write $u^{*}(x)=\left\lfloor u^{*}(x)\right\rfloor+b$ with $0<b<1$, where $\left\lfloor u^{*}(x)\right\rfloor$ is the largest integer smaller than $u^{*}(x)$ (i.e. the floor function). Performing the change of variable $m=k-\left\lfloor u^{*}(x)\right\rfloor$ in the discrete sum (16), such that $k-u^{*}(x)=m-b$ one obtains the useful expression

$$
\begin{aligned}
& \sum_{k=0}^{n} g\left(k-u^{*}(x)\right) \exp (-\phi(k, x)) \\
& \sim e^{-\phi\left(u^{*}(x), x\right)} \sum_{m=-\left\lfloor u^{*}(x)\right\rfloor}^{n-\left\lfloor u^{*}(x)\right\rfloor} g(m-b) \exp \left[-\frac{\alpha(\alpha-1)}{2}(m-b)^{2}\left[u^{*}(x)\right]^{\alpha-2}\right] .
\end{aligned}
$$

One clearly sees in expression (18) that the behavior of this discrete sum, due to the term $\left[u^{*}(x)\right]^{\alpha-2} \propto(\log x)^{(\alpha-2) /(\alpha-1)}$, will depend on the sign of $\alpha-2$. We will thus treat the three cases $1<\alpha<2, \alpha>2$ and $\alpha=2$ separately. This will be done in section 4 , 5 and 6 respectively.

\section{The case $0<\alpha<1$}

In that case, from the expression for $c_{n}(x)$ in Eq. (13), we can use the results of Kac's polynomials to obtain that most of the real roots will be such that, for large $n$, $x e^{-\frac{\alpha}{2} n^{\alpha-1}}-1=\mathcal{O}\left(n^{-1}\right)[12$. In other words, the real roots are distributed in a region 
of width $1 / n$ around $e^{\frac{\alpha}{2} n^{\alpha-1}}=1+\frac{\alpha}{2} n^{\alpha-1}+\mathcal{O}\left(n^{\alpha-2}\right)$ and this distribution is exactly the same as the one for Kac's polynomials (corresponding to $\alpha=0$ ). The number of real roots is thus also the same and given by

$$
\left\langle N_{n}\right\rangle \sim \frac{2}{\pi} \log n,
$$

independently of $\alpha<1$.

\section{The case $1<\alpha<2$}

In that case $\left[u^{*}(x)\right]^{\alpha-2} \rightarrow 0$ for large $u^{*}(x)$ and one thus sees on the asymptotic expression in Eq. (18) that the discrete sum can be replaced by an integral. This yields, for large $n$ and large $x$ with $x<\exp \left(\frac{\alpha}{2} n^{\alpha-1}\right)$

$$
\sum_{k=0}^{n} g\left(k-u^{*}(x)\right) \exp (-\phi(k, x)) \sim e^{-\phi\left(u^{*}(x), x\right)} \int_{-\infty}^{\infty} g(y) e^{-\frac{\alpha(\alpha-1)}{2} y^{2} u^{*}(x)^{\alpha-2}} d y .
$$

Note that the prefactor $e^{-\phi\left(u^{*}(x), x\right)}$ is unimportant for the computation of $\rho_{n}(x)$ because it disappears between the numerator and the denominator in Eq. (14) and it will be omitted below. In particular, setting $g(z)=1$ in Eq. (19) one has

$$
\sum_{k=0}^{n} \exp (-\phi(k, x)) \propto \sqrt{2 \pi}\left[\frac{u^{*}(x)^{2-\alpha}}{\alpha(\alpha-1)}\right]^{\frac{1}{2}}
$$

and similarly, setting $g(z)=z^{2}$ in Eq. (19) one has

$$
\sum_{k=0}^{n}\left(k-u^{*}(x)\right)^{2} \exp (-\phi(k, x)) \propto \sqrt{2 \pi}\left[\frac{u^{*}(x)^{2-\alpha}}{\alpha(\alpha-1)}\right]^{\frac{3}{2}},
$$

while $\sum_{k=0}^{n}\left(k-u^{*}(x)\right) \exp (-\phi(k, x)) \sim 0$ to lowest order in $n$. Therefore using the exact expression given in Eq. (14) together with the asymptotic behaviors given in Eq. (20, 21), one obtains the large $x$ behavior of $\rho_{n}(x)$ as

$$
\rho_{n}(x) \sim \frac{1}{\pi x} \frac{1}{\sqrt{\alpha(\alpha-1)}}\left(\frac{2}{\alpha} \log x\right)^{\frac{2-\alpha}{2(\alpha-1)}} .
$$

For a clear comparison with the case $\alpha>2$ (which will be analysed in the next section), it is convenient to write the density $\hat{\rho}_{n}(Y)$, in terms of the variable $Y=\left(\frac{2}{\alpha} \log x\right)^{\frac{1}{\alpha-1}}$, which reads, for $1 \ll Y<n$

$$
\hat{\rho}_{n}(Y) \sim \frac{\sqrt{\alpha(\alpha-1)}}{2 \pi} Y^{-\frac{1}{2}(2-\alpha)}
$$

and in Fig. 2 a), we show a sketch of this asymptotic behavior (23) of $\hat{\rho}_{n}(Y)$ for $1 \ll Y<n$.

We can now compute $\left\langle N_{n}\right\rangle=\int_{-\infty}^{\infty} \rho_{n}(x) d x$. First, one notices that for $\alpha>1$, the series entering in the definition of $c_{n}(x)$ in Eq. (10) has an infinite radius of convergence 
so that one readily obtains that $\int_{-1}^{+1} \rho_{n}(x) d x$ is of order $\mathcal{O}(1)$ in the limit $n \rightarrow \infty$. Besides, for large $x \gg e^{\frac{\alpha}{2} n^{\alpha-1}}$, one has (see also Ref. [7])

$$
\rho_{n}(x) \sim \sqrt{\frac{\left\langle a_{n-1}^{2}\right\rangle}{\left\langle a_{n}^{2}\right\rangle}} \frac{1}{\pi x^{2}} \sim \frac{e^{\frac{\alpha}{2} n^{\alpha-1}}}{\pi x^{2}},
$$

which implies that $\int_{e^{\frac{\alpha}{2} n^{\alpha-1}}}^{\infty} \rho_{n}(x) d x$ is also of order $\mathcal{O}(1)$ in the limit $n \rightarrow \infty$. From these properties, it follows that the main contributions to $\left\langle N_{n}\right\rangle$ on $\mathbb{R}^{+}$comes from the interval $\left[1, e^{\frac{\alpha}{2} n^{\alpha-1}}\right]$ where the asymptotic behavior of $\rho_{n}(x)$ is given in Eq. (22). Therefore one has

$$
\left\langle N_{n}\right\rangle \sim 2 \int_{1}^{e^{\frac{\alpha}{2} n^{\alpha-1}}} \rho_{n}(x) d x \sim \frac{2}{\pi} \sqrt{\frac{\alpha-1}{\alpha}} n^{\alpha / 2},
$$

where the factor 2 comes from the additional contribution coming from $\left[-e^{\frac{\alpha}{2} n^{\alpha-1}},-1\right]$. We thus have here an algebraic growth $\left\langle N_{n}\right\rangle \propto n^{\alpha / 2}$ with a continuously varying exponent $\alpha / 2$. This exponent tends to $1 / 2$ as $\alpha \rightarrow 1^{+}$, which is expected from the analysis of Weyl polynomials $W_{n}(x)$ in Eq. (1) for which $\left\langle a_{k}^{2}\right\rangle \propto e^{-k \log k}$ (although the variance is not exactly of the form $\left\langle a_{k}^{2}\right\rangle=e^{-k^{\alpha}}$ ). Besides, from Eq. (25), one also obtains that the amplitude of this term proportional to $n^{\alpha / 2}$ vanishes when $\alpha \rightarrow 1$. We recall that for $\alpha \leq 1$, one has instead $\left\langle N_{n}\right\rangle \propto\left(\frac{2}{\pi}\right) \log n$ (18), characteristic for Kac's polynomials. This suggests that this limit $\alpha \rightarrow 1$ is rather singular in the sense that the asymptotic behavior of $\left\langle N_{n}\right\rangle$ for large $n$ changes "discontinuously" from $\log n$ to $\sqrt{n}$.

\section{The case $\alpha>2$}

In that case, the behavior of the discrete sum in Eq. (18), which enters in the computation of $\rho_{n}(x)(14)$ is quite different. Indeed, in that case $\left[u^{*}(x)\right]^{\alpha-2} \propto$ $(\log x)^{(\alpha-2) /(\alpha-1)} \rightarrow \infty$ for large $x$ and therefore the leading term for large $x$ in Eq. (18) corresponds to $m=0$ if $b<1 / 2$ or $m=1$ in $b>1 / 2$. Keeping these leading contributions, one has

$$
\begin{aligned}
& \sum_{k=0}^{n} g\left(k-u^{*}(x)\right) \exp (-\phi(k, x)) \propto g(-b) \exp \left[-\frac{\alpha(\alpha-1)}{2} b^{2} u^{*}(x)^{\alpha-2}\right] \\
& +g(1-b) \exp \left[-\frac{\alpha(\alpha-1)}{2}(1-b)^{2} u^{*}(x)^{\alpha-2}\right] .
\end{aligned}
$$

where, again, we have omitted the unimportant prefactor $e^{-\phi\left(u^{*}(x), x\right)}$. Using this large $x$ expansion (26) , one obtains $\rho_{n}(x)$ in Eq. (14) as

$$
\rho_{n}(x) \sim \frac{2}{(\pi x) \cosh \left[\frac{\alpha(\alpha-1)}{2} Y^{\alpha-2}(1-2 b)\right]}, Y=\left(\frac{2}{\alpha} \log x\right)^{\frac{1}{\alpha-1}} .
$$

In terms of the variable $Y$, the density $\hat{\rho}_{n}(Y)$ reads,

$$
\hat{\rho}_{n}(Y=\lfloor Y\rfloor+b) \sim \frac{\alpha(\alpha-1) Y^{\alpha-2}}{2 \pi \cosh \left[\frac{\alpha(\alpha-1)}{2} Y^{\alpha-2}(1-2 b)\right]} .
$$


In Fig. 2 c), one shows a sketch of $\hat{\rho}_{n}(Y)$ for large $Y<n$ given by Eq. (28) : it is qualitatively very different from the case $\alpha<2$ (see Fig. 2] a)). Indeed, $\hat{\rho}_{n}(Y)$ exhibits peaks centered around $k+\frac{1}{2}$ for large integers $1 \ll k<n$. The height of these peaks is given by $\alpha(\alpha-1) k^{\alpha-2} /(2 \pi)$ whereas its width scales like $k^{2-\alpha}$.

From $\rho_{n}(x)$, one can now compute the mean number of real roots. As in the case $\alpha<2$ (see Eq. (24) and above), one can show that the main contribution to $\left\langle N_{n}\right\rangle$ comes from the intervals $\left[-e^{\frac{\alpha}{2} n^{\alpha-1}},-1\right]$ and $\left[1, e^{\frac{\alpha}{2} n^{\alpha-1}}\right]$. One thus has from Eq. (28)

$$
\begin{aligned}
\left\langle N_{n}\right\rangle & =2 \int_{0}^{\infty} \rho_{n}(x) d x \sim 2 \int_{0}^{n} \hat{\rho}_{n}(Y) d Y \\
& \sim \sum_{k \gg 1}^{n} \int_{0}^{1} \frac{\alpha(\alpha-1) k^{\alpha-2}}{\pi \cosh \left[\frac{\alpha(\alpha-1)}{2} k^{\alpha-2}(1-2 b)\right]} d b \sim \sum_{k \gg 1}^{n} \int_{-\frac{\alpha(\alpha-1)}{2} k^{\alpha-2}}^{\frac{\alpha(\alpha-1)}{2} k^{\alpha-2}} \frac{d z}{\pi \cosh z},
\end{aligned}
$$

and finally

$$
\left\langle N_{n}\right\rangle \sim n,
$$

where we have used $\int_{-\infty}^{\infty} d z / \cosh z=\pi$. This condensation of the roots on the real axis, characterized by the fact that $\left\langle N_{n}\right\rangle \sim n$ thus occurs via the formation of this quasi-periodic structure (see Fig. 2 c)). More precisely, this computation in Eq. (29) shows that for large $k, 2 \int_{k}^{k+1} \hat{\rho}_{n}(Y) d Y \sim 1$ which means, going back to the original variable $x$, that there is, on average, one root in the interval $\left[-x_{k+1},-x_{k}\right] \cup\left[x_{k}, x_{k+1}\right]$, with $x_{k}=e^{\frac{\alpha}{2} k^{\alpha-1}}$.

\section{The special case $\alpha=2$}

In view of the previous analysis, it is tempting to consider the fraction of real roots $\Phi=\lim _{n \rightarrow \infty}\left\langle N_{n}\right\rangle / n$ as an "order paramater". For $\alpha<2$, one has $\Phi=0$ whereas $\Phi=1$ for $\alpha>2$. One can however interpolate smoothly between these two limiting cases by considering the case $\alpha=2$ and introducing an additional real parameter $\mu$ such that

$$
\left\langle a_{k}^{2}\right\rangle=e^{-\mu k^{2}} .
$$

Performing the same algebra as explained in the Appendix, one obtains the same formula as given in Eq. (14) with $u^{*}(x)=\mu^{-1} \log x$. The new variable is thus here $Y=\mu^{-1} \log x$ and, setting $Y=\lfloor Y\rfloor+b$ it is easy to see that the density $\hat{\rho}_{n}(Y)$ is given by for $1 \ll Y<n$

$$
\hat{\rho}_{n}(Y)=\frac{\mu}{\pi}\left[\frac{\sum_{m=-\infty}^{\infty}(m-b)^{2} e^{-\mu(m-b)^{2}}}{\sum_{m=-\infty}^{\infty} e^{-\mu(m-b)^{2}}}-\left[\frac{\sum_{m=-\infty}^{\infty}(m-b) e^{-\mu(m-b)^{2}}}{\sum_{m=-\infty}^{\infty} e^{-\mu(m-b)^{2}}}\right]^{2}\right]^{1 / 2},
$$

which is thus 1-periodic for all $\mu$. In Fig. 2 c), one shows a sketch of $\hat{\rho}(Y)$ for $\alpha=2$ given by Eq. (32). For $\mu \rightarrow 0$, the density is almost constant and $\hat{\rho}_{n}(Y) \sim \pi^{-1} \sqrt{\mu / 2}$ and the modulation of the density increases with $\mu$. For large $\mu$, the sum in Eq. (32) is dominated by the terms corresponding to $m=0$ and $m=1$ and $\hat{\rho}_{n}(Y)$ is thus given by a formula similar to Eq. (28) setting $\alpha=2$ and replacing $Y^{\alpha-2}$ by $\mu$. For the average 

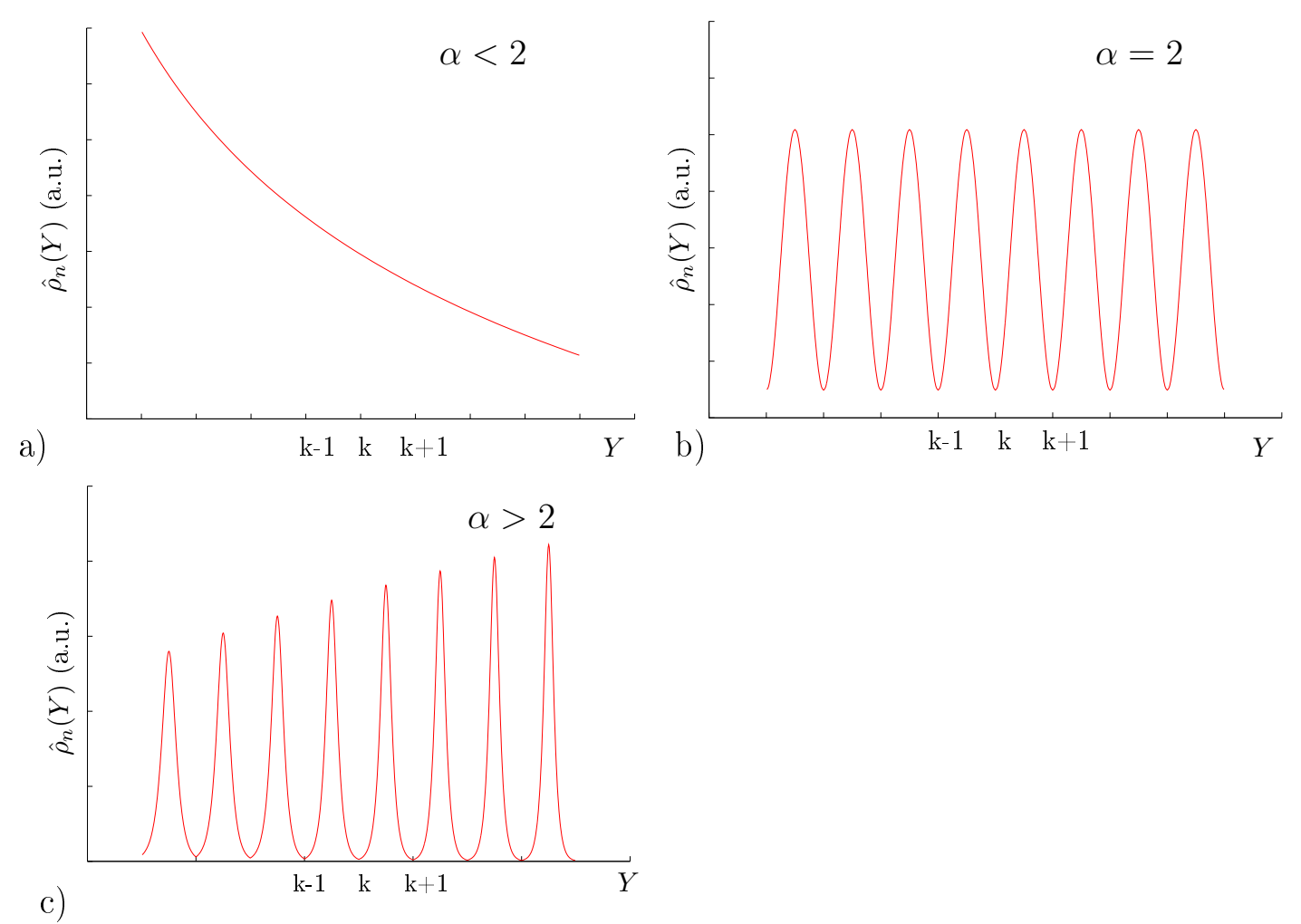

Figure 2. a) : Sketch of $\hat{\rho}_{n}(Y)$ (in arbitrary units) given in Eq. (23) as a function of $Y$ for $1 \ll Y<n$ for $\alpha<2$. b) : Sketch of $\hat{\rho}_{n}(Y)$ (in arbitrary units) given in Eq. (32) as a function of $Y$ for $1 \ll Y<n$ for $\alpha=2$. c) : Sketch of $\hat{\rho}_{n}(Y)$ (in arbitrary units) given in Eq. (28) as a function of $Y$ for $1 \ll Y<n$ for $\alpha>2$. Here $k$ denotes an integer with $1 \ll k<n$.

number of real roots one has

$$
\left\langle N_{n}\right\rangle \propto\left\{\begin{array}{l}
\frac{\sqrt{2 \mu}}{\pi} n, \mu \ll 1 \\
n, \mu \gg 1
\end{array}\right.
$$

which shows that this family of real random polynomials (31) interpolate smoothly between the cases $\alpha<2(\underline{25})$ and $\alpha>2(\underline{29})$.

\section{A qualitative argument for the transition at $\alpha=2$}

This condensation of the roots on the real axis can be qualitatively understood if one considers the random polynomials (for $x>0) \hat{P}_{n}(Y)=P_{n}(x)$ of the variable $Y$, which one writes as

$$
\hat{P}_{n}(Y)=\sum_{k=0}^{n} \hat{a}_{k} w(k, Y), w(k, Y)=\exp \left[-\frac{1}{2}\left(k^{\alpha}-\alpha k Y^{\alpha-1}\right)\right],
$$

and $\hat{a}_{k}$ are i.i.d. Gaussian variables of unit variance. It is easy to see that the weights $w(k, Y)$, as a function of $k$, have a single maximum for $k=Y$ where the second derivative is proportional to $k^{\alpha-2}$. Thus for $\alpha>2$, the weights get more and more peaked around 
this maximum for large $k$, whereas $\hat{a}_{k}$ is typically of order $\mathcal{O}(1)$. Therefore, given a large integer $m, \hat{P}_{n}(m)$ is, for $\alpha>2$, dominated by a single term corresponding to $k=m$. Consequently, the sign of $\hat{P}_{n}(m)$ is essentially the sign of $\hat{a}_{m}$. This in turn implies that, if $\hat{a}_{m}$ and $\hat{a}_{m+1}$ have an opposite sign, $P_{n}(x)$ has, with a probability close to 1 , a root in the interval $\left[e^{\frac{\alpha}{2} m^{\alpha-1}}, e^{\frac{\alpha}{2}(m+1)^{\alpha-1}}\right]$. In the case where $\hat{a}_{m}$ and $\hat{a}_{m+1}$ have the same sign, the same argument shows that $P_{n}(x)$ has, with a probability close to 1 , a root in the interval $\left[-e^{\frac{\alpha}{2}(m+1)^{\alpha-1}},-e^{\frac{\alpha}{2}(m)^{\alpha-1}}\right]$. One thus recovers qualitatively the result we had found from the computation of $\hat{\rho}_{n}(Y)$ in Eq. (29) where we have shown that $P_{n}(x)$ has, on average, one root in the interval $\left[-e^{\frac{\alpha}{2}(m+1)^{\alpha-1}},-e^{\frac{\alpha}{2}(m)^{\alpha-1}}\right] \cup\left[e^{\frac{\alpha}{2} m^{\alpha-1}}, e^{\frac{\alpha}{2}(m+1)^{\alpha-1}}\right]$. This shows finally that $P_{n}(x)$ has, on average, $\left\langle N_{n}\right\rangle \propto n$ real roots.

We also point out that our argument explains in a rather intuitive way the result obtained by Littlewood and Offord [11] for the random polynomials $L_{n}(x)$ (2). For these specific polynomials, defining $x_{0}=0, x_{m}=m^{m} m$ !, they rigorously proved, using

algebraic (and rather cumbersome) methods, that $L_{n}(x)$ has a root either on $\left[x_{m}, x_{m+1}\right]$ if $\epsilon_{m} \epsilon_{m+1}=-1$ or in $\left[-x_{m+1},-x_{m}\right]$ if $\epsilon_{m} \epsilon_{m+1}=1$. Our argument gives some insight on their intriguing result and allows to understand it in a rather simple way.

\section{Conclusion}

To conclude we have introduced a new family of random polynomials (3)), indexed by a real $\alpha$. For these random polynomials, we have computed the mean density of real roots $\rho_{n}(x)$ from which we computed the mean number of real roots $\left\langle N_{n}\right\rangle$ for large $n$. We have shown that, while for $0 \leq \alpha<1,\left\langle N_{n}\right\rangle \sim\left(\frac{2}{\pi}\right) \log n$, the behavior of $\left\langle N_{n}\right\rangle$ for $\alpha>1$ deviates significantly from the logarithmic behavior characteristic for Kac's polynomials. For $1<\alpha<2$, we have shown that $\left\langle N_{n}\right\rangle \propto n^{\alpha / 2}$ whereas for $\alpha>2,\left\langle N_{n}\right\rangle \sim n$. This family of real random polynomials thus displays an interesting condensation phenomenon of their roots on the real axis, which is accompanied by an ordering of the roots in a quasi periodic structure : this is depicted in Fig. 2 ,

Of course, the occurrence of this transition raises several interesting questions like the behavior of the variance of the number of real roots for large $n$ as $\alpha$ is varied. It would be also interesting to compute the two-point correlation function of the real roots, which is a rather natural tool to characterize this periodic structure we have found. In view of this, we hope that this interesting phenomenon will stimulate further research on random polynomials.

\section{Appendix A. A useful expression for the mean density $\rho_{n}(x)$}

In this appendix, we derive the expression for the mean density $\rho_{n}(x)$ as given in Eq. (14) starting from Eq. (7). We first write $c_{n}(x)=\left\langle P_{n}(x) P_{n}(x)\right\rangle$ as

$$
c_{n}(x)=e^{-\phi\left(u^{*}(x), x\right)} \sum_{k=0}^{n} e^{-\tilde{\phi}(k, x)},
$$


where $u^{*}(x)$ is the location of the minimum of $\phi(u, x)$ given in Eq. (11)

$$
u^{*}(x)=\left(\frac{2}{\alpha} \log x\right)^{\frac{1}{\alpha-1}}
$$

and

$$
\begin{aligned}
& \phi\left(u^{*}(x), x\right)=(1-\alpha) u^{*}(x)^{\alpha} \\
& \tilde{\phi}(k, x)=\phi(k, x)-\phi\left(u^{*}(x), x\right)=k^{\alpha}-\alpha k\left[u^{*}(x)\right]^{\alpha-1}+(\alpha-1)\left[u^{*}(x)\right]^{\alpha} .
\end{aligned}
$$

The correlator $C_{n}(x, y)=c_{n}(\sqrt{x y})$ is given by Eq. (A.1) together with Eq. (A.3) where $x$ is replaced by $\sqrt{x y}$. All the dependence of $C_{n}(x, y)$ in $x, y$ is thus contained in $u^{*}(\sqrt{x y})$ only. From its definition in Eq. (A.2) one has immediately

$$
\partial_{x} u^{*}(\sqrt{x y})=\frac{1}{\alpha(\alpha-1)} \frac{1}{x}\left[u^{*}(\sqrt{x y})\right]^{2-\alpha},
$$

from which we obtain a set of useful relations

$$
\begin{aligned}
& \partial_{x, y}^{2} \phi\left(u^{*}(\sqrt{x y}), \sqrt{x y}\right)=-\frac{1}{\alpha(\alpha-1)} \frac{1}{x y}\left[u^{*}(\sqrt{x y})\right]^{2-\alpha} \\
& \partial_{x} \tilde{\phi}(k, \sqrt{x y})=\frac{1}{x}\left(u^{*}(\sqrt{x y})-k\right) \\
& \partial_{x, y}^{2} \tilde{\phi}(k, \sqrt{x y})=\frac{1}{\alpha(\alpha-1)} \frac{1}{x y}\left[u^{*}(\sqrt{x y})\right]^{\alpha-2} .
\end{aligned}
$$

For the computation of $\rho_{n}(x)$ from Eq. (7), it is useful to introduce the notation, for any function $g(k)$

$$
\langle g(k)\rangle_{Z}=\frac{\sum_{k=0}^{n} g(k) \exp (-\tilde{\phi}(k, \sqrt{x y}))}{\sum_{k=0}^{n} \exp (-\tilde{\phi}(k, \sqrt{x y}))} .
$$

From $C_{n}(x, y)=c_{n}(\sqrt{x y})$ and $c_{n}(x)$ given in Eq. A.1 $)$ one obtains

$$
\begin{aligned}
& \partial_{x} \partial_{y} \log C_{n}(\sqrt{x y})=-\partial_{x, y}^{2} \phi\left(u^{*}(\sqrt{x y}), \sqrt{x y}\right)-\left\langle\partial_{x} \tilde{\phi}(k, \sqrt{x y}) \partial_{y} \tilde{\phi}(k, \sqrt{x y})\right\rangle_{Z} \\
& -\left\langle\partial_{x} \tilde{\phi}(k, \sqrt{x y})\right\rangle_{Z}\left\langle\partial_{x} \tilde{\phi}(k, \sqrt{x y})\right\rangle_{Z}-\left\langle\partial_{x, y}^{2} \tilde{\phi}(k, \sqrt{x y})\right\rangle_{Z} .
\end{aligned}
$$

From the above relations in Eq. (A.5), it is readily seen that the first and the last term in Eq. (A.7) cancel each other. Using the relation in Eq. (7), one finally obtains the relation given in the text in Eq. (14).

\section{References}

[1] A. Bloch and G. Pólya, On the roots of certain algebraic equations, Proc. London Math. Soc. (3) 33, 102 (1932).

[2] A. T. Bharucha-Reid and M. Sambandham, Random Polynomials, Academic Press, New York, 1986.

[3] K. Farahmand, in Topics in random polynomials, Pitman research notes in mathematics series 393, (Longman, Harlow) (1998).

[4] A. Edelman and E. Kostlan, How many zeros of random polynomials are real ?, Bull. Amer. Math. Soc. 32, 1 (1995).

[5] E. Bogomolny, O. Bohigas and P. Leboeuf, Distribution of roots of random polynomials Phys. Rev. Lett. 68, 2726 (1992); Quantum chaotic dynamics and random polynomials, J. Stat. Phys. 85, 639. (1996). 
[6] G. Schehr, S.N. Majumdar, Statistics of the number of zero crossings: from random polynomials to the diffusion equation, Phys. Rev. Lett. 99, 060603 (2007).

[7] G. Schehr, S.N. Majumdar, J. Stat. Phys. 132, 235 (2008).

[8] M. Kac, On the average number of real roots of a random algebraic equation, Bull. Amer. Math. Soc. 49, 314 (1943); Erratum: Bull. Amer. Math. Soc. 49, 938 (1943).

[9] M. Das, Real zeros of a class of random algebraic polynomials, J. Indian Math. Soc. 36, 53 (1972).

[10] P. Leboeuf, Random Analytic Chaotic Eigenstates, J. Stat. Phys. 95, 651 (1999).

[11] J.E. Littlewood and A.C. Offord, On the number of real roots of a random algebraic equation. II, Proc. Cambridge Phil. Soc. 35, 133 (1939).

[12] A.P. Aldous and Y.V. Fyodorov, Real roots of random polynomials: universality close to accumulation points, J. Phys. A: Math. Gen. 37, 1231 (2004) 